\title{
Transient Proximal Flow Arrest Technique for Crossing Giant Aneurysm
}

\author{
Sea Mi Park and Daniel Walzman*
}

North Jersey Brain and Spine Center, USA

ISSN: 2637-7748

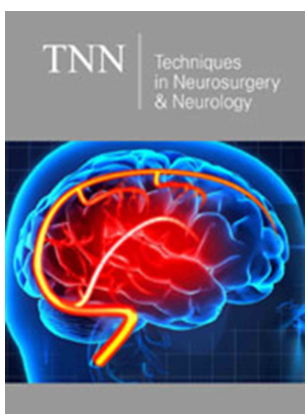

*Corresponding author: Daniel Walzman, North Jersey Brain and Spine Center, USA

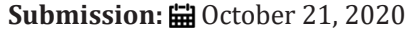

Published: 僵 November 20, 2020

Volume 3 - Issue 4

How to cite this article: Sea Mi Park, Daniel Walzman. Transient Proximal Flow Arrest Technique for Crossing Giant Aneurysm. Tech Neurosurg Neurol. 3(4). TNN. 000568. 2020.

DOI: $10.31031 /$ TNN.2020.03.000568

Copyright@ Daniel Walzman, Gonzalez EKB and Esqueda LMA, This article is distributed under the terms of the Creative Commons Attribution 4.0 International License, which permits unrestricted use and redistribution provided that the original author and source are credited.

\section{Introduction}

A giant intracranial aneurysm is known to have a natural history of high rupture rate which leads to significant morbidity, mortality, and health care costs [1,2]. High complication rate has been reported with both surgical and endovascular treatment [3-5]. Pipeline embolization device (PED) use in giant intracranial aneurysms has become increasingly established for treatment in many of these cases as retrospective and prospective studies demonstrates lower overall procedural cost and higher efficacy and safety protecting the parent artery $[6,7]$. However, the treatment of giant aneurysms remains a formidable challenge due to technical aspect of PED placement. Giant aneurysms could have strong inflow jets, which is reported a link between vertical flow and rupture status as well as technical challenge to direct microcatheters over wires into the parent artery across an aneurysmal. In many cases, this procedural step can be prohibitively difficult due to the aneurysm inflow jet sucking microcatheter over wires into the aneurysm and precluding access to the parent artery distal to the aneurysm. We report on the first case of a transient proximal flow arrest technique to prevent inflow jets in order to cross the neck of a giant intracranial aneurysm to facilitate PED placement.

\section{Case Report}

A 49-year-old female who presented with progressive blurry vision referable to a giant saccular aneurysm in ophthalmic segment of internal carotid artery (ICA) without evidence of aneurysmal rupture. Initial non-invasive neuroimaging and subsequent catheter cerebral angiography identified a giant aneurysm measuring $3.56 \mathrm{~mm} \times 2.6 \mathrm{~mm}$ in maximal diameter arising distally from ophthalmic artery given the size and location of the aneurysm, the decision was made to treat the patient using an endovascular approach using the Pipeline Embolization Device (PED, ev3-Covidien, Plymouth, MN, USA). The patient was premedicated with aspirin $325 \mathrm{mg}$ daily and Plavix $75 \mathrm{mg}$ daily for 7 days prior to undergoing the stent procedure. Result of the patient's Plavix assay showed therapeutic level (Verify Now P2Y12 assay=78) on the day of procedure. Prior to procedure, the patient as treated with 2 grams of Ancef and 10 grams of Decadron. Under general anesthesia, femoral sheath was place in right femoral artery after needle puncture. 5-Fr diagnostic catheter was positioned in left lower cervical ICA and performed 3-D rotational angiography to determine working projection. Under roadmap guidance, diagnostic catheter was exchanged for 8-FrFlowGate (Stryker Neurovascular, Fremont, CA) guide catheter through which NavienTMIntracranial

Support Catheter (0.058", ev3-Covidien, Mansfield, MA, USA) was advanced over a PhenomTMmicrocatheter (0.027" Medtronic, Minneapolis, MN) over a Fathom microwire (0.016" Boston Scientific, Marlborough MA).

There was deflection into the aneurysm upon initial attempts to cross the aneurysm neck, therefore, the Flow Gate balloon was inflating within proximal left ICA temporarily arresting flow. With flow arrested in the ICA, the aneurysmal neck was easily crossed without deflection due to effect of inflow jets in the neck. Flow was restored after 30seconds while Phenom catheter positioned within the M1 segment of left middle cerebral artery (MCA). Follow up super selective angiogram of the left MCA distribution demonstrated an appropriate placement of distal tip of Phenom microcatheter. Next, the first PED $(3.75 \mathrm{~mm}$ by $16 \mathrm{~mm})$ was advanced 
through the Phenom catheter, then, deployed in a standard fashion and opened at the level of the ICA terminus, and landed proximal from aneurysmal neck. Post PED control angiogram of left ICA demonstrated patent PED without evidence of thromboembolic complication (Figures 1-3).

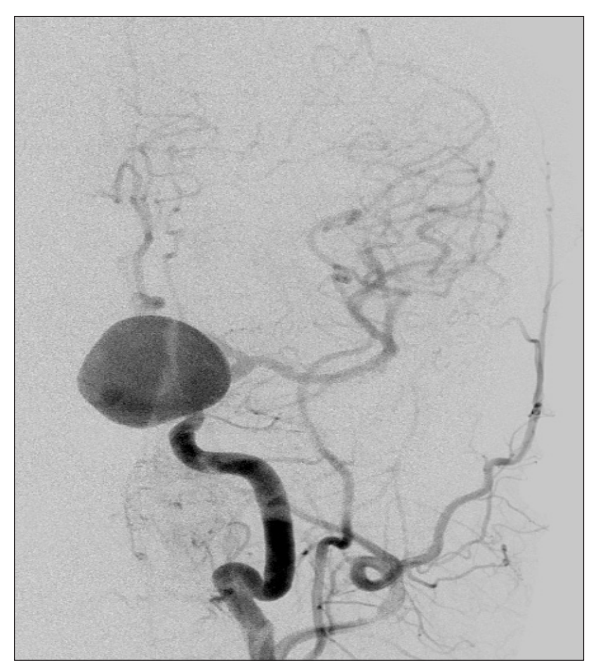

Figure 1: Origin which is directing medioposteriorly.

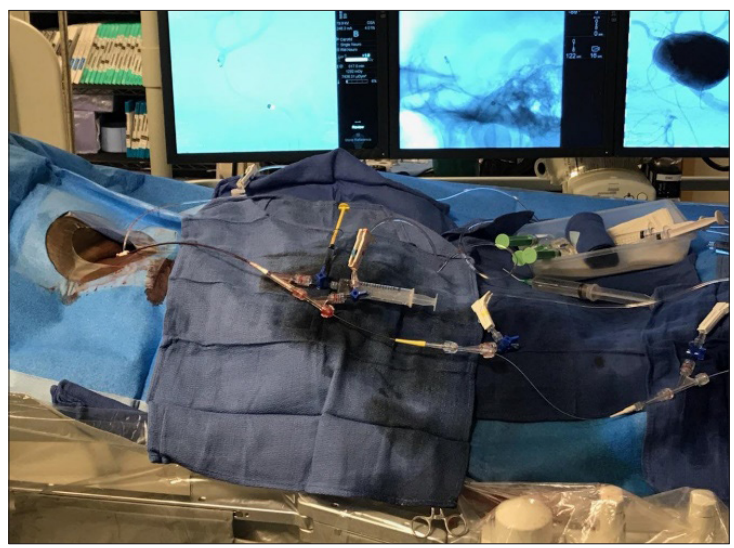

Figure 2.

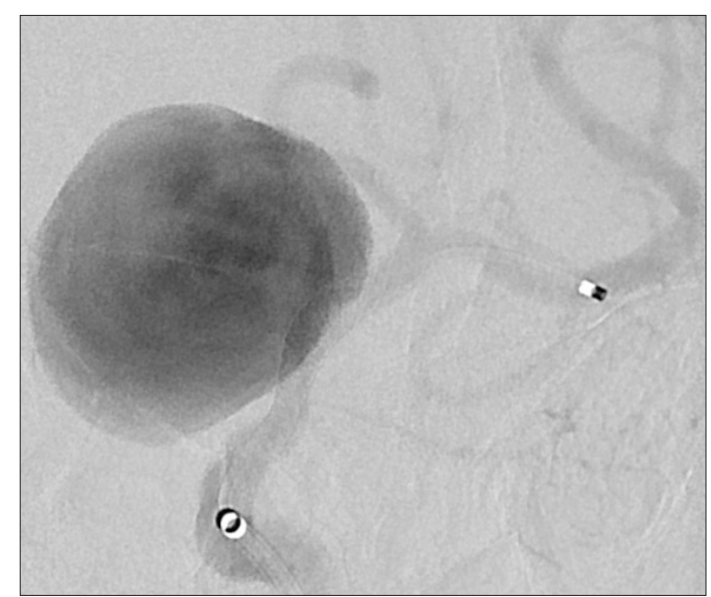

Figure 3.
Of note, the inflow jets into the aneurysm are moderately diminished. After first post-PED control angiogram decision was made to place second PED placement to secure the PED construction across the aneurysmal neck. The Phenom catheter was again re-positioned within left MCA M1 segment and the second PED (5mmX16mm) device was advanced through Phenom microcatheter. This was deployed in a telescoping fashion, landing distally within the previously placed device, and extending across the neck of the aneurysm into the cavernous segment of left ICA. Second post-PED control angiogram demonstrated appropriate positioning of the PED without evidence of thromboembolic complication (Figure 4). The inflow jets into the aneurysm were further decreased with contrast stasis in ten minutes delayed follow up angiogram (Figure 5). The patient's post procedural course was uneventful. She was discharged home 3 days after the procedure without neurological deficit with continuation of dual antiplatelet therapy. The patient will continue to be monitored with serial angiography for progressive aneurysm thrombosis (Figure 6).

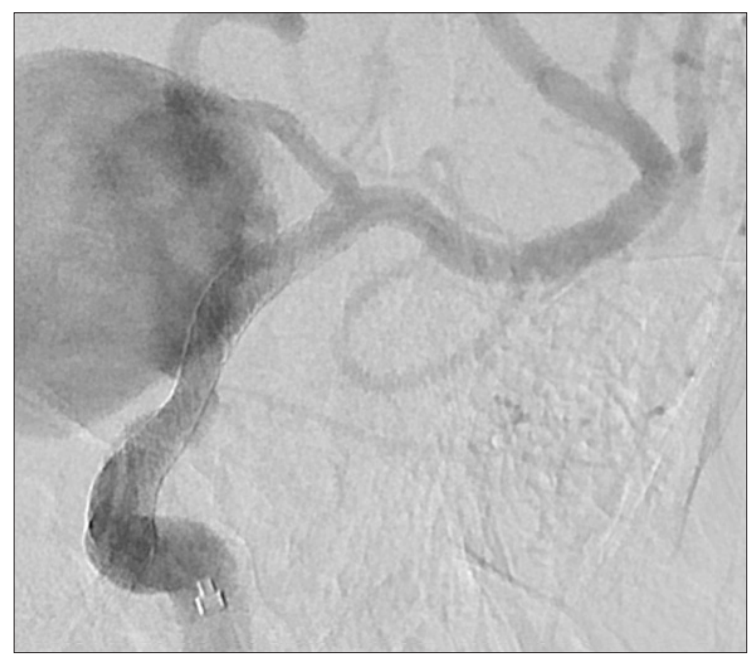

Figure 4.

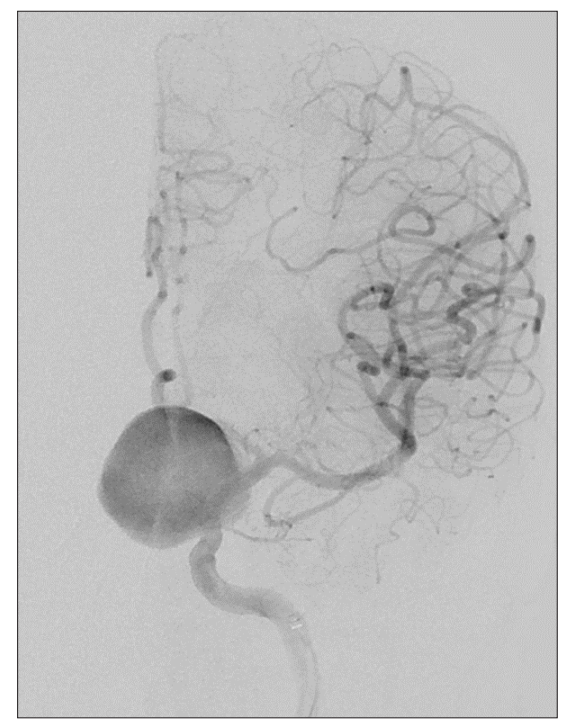

Figure 5. 


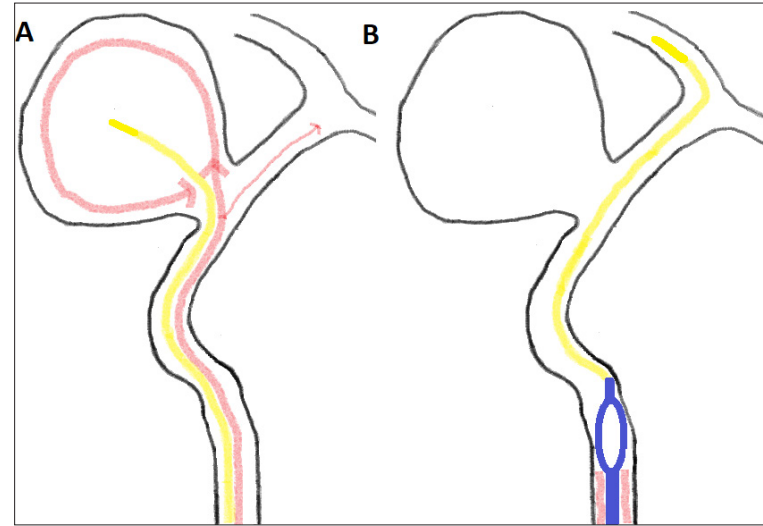

Figure 6: (A) Illustration of aneurysm flow modes based on the side wall inflow jet in giant ICA aneurysm: inflow jet is sucking microcatheter into the aneurysm, (B) Mechanism of Transient Proximal Flow Arrest Technique using proximal balloon guide catheter Red-Blood flow, Yellowmicrocatheter, Blue-Balloon Catheter).

\section{Discussion}

Most recently, there has been a significant shift in the management of unruptured aneurysm away from surgery toward endovascular techniques with technical development of flow diverting device especially for the treatment of large aneurysms in ICA. PED provide powerful therapeutic efficiency and safety if it is technically feasible to deploy across the neck of the aneurysm. We reported first successful technical report of a novel transient proximal flow arrest technique for crossing giant intracranial aneurysms to facilitate deployment of a stent or PED across the aneurysm neck to increase PED applicability. Use of the flow arrest technique can prevent microcatheter deflection due to inflow jets into aneurysm in order to access the distal patent artery. Previous reported stent/balloon anchor technique [8-10] could help to prevent microcatheter redundancy when crossing the aneurysmal neck, however, temporally arrest of flow may be crucial strategy to prevent the mechanism of microcatheter deflection due to inflow jets into giant aneurysm. We currently report achieving such flow arrest via use of a proximal balloon guide catheter. Other technique to arrest flow, such as a balloon alongside the microcatheter (proximal to the aneurysm neck) or temporally cardiac arrest with adenosine may achieve a similar result as well.

\section{References}

1. Wiebers DO, Whisnant JP, Huston J, Meissner I, Brown RD, et al. (2003) Unruptured intracranial aneurysms: natural history, clinical outcome, and risks of surgical and endovascular treatment. Lancet 362(9378): 103-110.

2. Ogilvy CS, Carter BS (1998) A proposed comprehensive grading system to predict outcome for surgical management of intracranial aneurysms. Neurosurgery 42(5): 959-968.

3. Cantore G, Santoro A, Guidetti G, Delfinis CP, Colonnese C, et al. (2008) Surgical treatment of giant intracranial aneurysms: current viewpoint. Neurosurgery 63(4): 279-289.

4. Lawton MT, Quinones HA, Sanai N, Malek JY, Dowd CF (2008) Combined microsurgical and endovascular management of complex intracranial aneurysms. Neurosurgery 62(6): 1503-1515.

5. Maingard J, Brooks M (2016) Donut' basilar aneurysm with brainstem compression: treatment using a flow diverting stent. Interv Neuroradiol 22(3): 266-269.

6. Wali AR, Park CC, Santiago DDR, Vaida F, Murphy JD, et al. (2017) Pipeline embolization device versus coiling for the treatment of large and giant unruptured intracranial aneurysms: a cost-effectiveness analysis. Neurosurgical Focus 42(6): E6.

7. Becske T, Potts MB, Shapiro M, Kallmes DF, Brinjikji W, et al. (2017) Pipeline for uncoilable or failed aneurysms: 3-year follow-up results. J Neurosurg 127(1): 81-88.

8. Fargen KM, Velat GJ, Lawson MF, Hoh BL, Mocco J (2013) The stent anchor technique for distal access through a large or giant aneurysm. J Neurointerv Surg 5(4): e24.

9. Snyder KV, Natarajan SK, Hauck EF, Mocco J, Siddiqui AH, et al. (2010) The balloon anchor technique: a novel technique for distal access through a giant aneurysm. J Neurointerv Surg 2(4): 363-367.

10. Ding D, Starke RM, Evans AJ, Jensen ME, Liu KC (2014) Balloon anchor technique for pipeline embolization device deployment across the neck of a giant intracranial aneurysm. J Cerebrovasc Endovasc Neurosurg 16(2): $125-130$ 